\title{
Zur Constitution der Chinaalkaloide
}

\author{
(IV. Mittheilung) \\ Das Chinidin \\ von \\ Dr, Julius Würstl. \\ Aus dem chemischen Institute der k. k. Universitäf Graz.
}

(Vorgelegt in der Sitzung am 24. Jănner 1889).

Das Chinidin $\mathrm{C}_{20} \mathrm{H}_{24} \mathrm{~N}_{2} \mathrm{O}_{2}$, von Hesse Conchinin benannt, ist mit dem Chinin isomer. Es gibt mit Chlor und Ammoniak dieselbe Reaction wie Chinin, und ähnelt diesem auch durch die blaue Fluoreszenz, die seine saueren Salze in wässeriger Lösung zeigen. Im folgenden sollen jene Daten zusammengestellt werden, die für die Constitution des Chinidins Wichtigkeit haben.

Skraup ${ }^{1}$ hat für Chinin und Chinidin nachgewiesen, dass beide Körper bei gelinder Oxydation mit Kaliumpermanganat unter Abspaltung von Ameisensäure nach der Gleichnng $\mathrm{C}_{20} \mathrm{H}_{24} \mathrm{~N}_{2} \mathrm{O}_{2}+\mathrm{O}_{4}=\mathrm{C}_{19} \mathrm{H}_{22} \mathrm{~N}_{2} \mathrm{O}_{4}+\mathrm{CH}_{2} \mathrm{O}_{2}$ Basen von der Formel $\mathrm{C}_{19} \mathrm{H}_{22} \mathrm{~N}_{2} \mathrm{O}_{4}$ liefern, und schliesst hieraus auf eine besondere und gleichartige Stellung eines der 20 Kohlenstoffatome in den Muttersubstanzen, dessen leichtere Abspaltung die Bildung der Ameisensäure bedingt.

Chinin sowohl, als dessen primäres Oxydationsproduct, das Chitenin, liefern bei Oxydation mit Chromsäure: Chininsäure und einen saueren Syrup. Unter analogen Verhältnissen zeigt Chinidin dieselben Zerfallsproducte, das ist wieder Chininsäure und einen saueren Syrup, über den im Nachfolgenden noch eingehender die Rede sein soll.

1 Annalen 199, 340-354.

Chemie-Heft Nr. 1. 
Aus dieser Thatsache geht hervor, dass das Chinidin, ebenso wie das Chinin ein Derivat des P-Methoxy-Chinolins sein muss, da die Chininsäure die $\gamma$ P.-Carbonsäure des letzteren ist.

Das Chinidin enthält also gerade wie das Chinin, und in derselben Stellung eine Methoxylgruppe, und ist der Chinolinring desselben in derselben Stellung mit dem anderen Theile des Moleküls verbunden, als es im Chinin der Fall ist.

NachHes s e ${ }^{1}$ lässt sich in das Chinidin gerade so wie in das Chinin ein Acetyl einführen, wonach das zweite Sauerstoffatom beider Alkaloide in Form einer Hydroxylgruppe vorhanden sein kann. Ob dies nun der Fall ist oder nicht, jedenfalls zeigt das berührte gleichartige Verhalten beider Alkaloide eine neue Ähnlichkeit, und ist weiterhin sicher, dass das durch Acetyl ersetzbare Wasserstoffatom in beiden Basen nicht in dem Chininsäure liefernden Theile enthalten sein kann.

Nach all diesen Thatsachen ist der Constitutionsunterschied zwischen Chinin und Chinidin bis nun ganz unbekannt, und man wird ihn in jenem Theile ihres Moleküls zu suchen haben, der bei der Oxydation nicht in Chininsäure übergeht und der nach Skraup's Ansicht den früher erwähnten saueren Syrup liefert.

Für den "Chininsyrup" hat Skraup ${ }^{2}$ den Nachweis erbracht, dass er Cincholoipon und Cincholoiponsäure enthält, also dieselben Körper, die von ihm als Oxydationsproducte des Cinchonins beschrieben wurden.

Ich setzte mir die Aufgabe, die Natur des aus dem Chinidin entstehenden saueren Syrup's, welcher wie ich noch einmal erwähne nur aus jenem Molekültheile entsteht, der nicht in Chininsäure übergeht, festzustellen.

\section{Oxydation des Chinidins.}

Es wurde Chinidinsulfat aus der Fabrik von Zimmer in Frankfurt verwendet, dessen Reinheit durch folgende Reactionen nachgewiesen worden ist. $0.50 \mathrm{~g}$ in $10 \mathrm{~cm}$ Wasser gelöst, wurden

1 Annal. 205, 318.

¿ Siehe die zweitvorhergehende Abhandlung dieses Heftes. 
mit dem gleichen Quantum von Judkalium versetzt, und eine halbe Stunde stehen gelassen; das Filtrat vom abgeschiedenen jodwasserstoffsaueren Chinidin blieb auf Zusatz eines Tropfen Ammoniaks vollkommen klar. Ferner gab ein Gramm des Salzes mit $7 \mathrm{~cm}$ einer Mischung von Alkohol mit Chloroform im Raumverhältniss 1 zu 2, klare Lösung. ${ }^{1}$

Da nach der bisherigen Erfahrung Cinchonin und Chinin bei directer Oxydation mit Chromsäure die Spaltungsproducte in weit schwieriger trennbarer Mischung liefern, als wenn man zuerst mit Kaliumpermanganat die phenolartigen, primären Oxydationsproducte (Cinchotenin, Chitenin etc.) darstellt, and diese dann weiter mit Chromsäure oxydirt, bin ich in ähnlicher Art vorgegangen.

Dementsprechend löste ich $200 \mathrm{~g}$ Chinidinum sulfuricum, die $154 \mathrm{~g}$ Base enthalten, mit $130 \mathrm{~g}$ concentritter Schwefelsäure in Wasser auf einen Liter und führte die Oxydation mit $4 \%$ Chamaeleonlösung in der Weise durch, dass zu je $50 \mathrm{~cm}$ der schwefelsaueren Lösung $315 \mathrm{~cm}$ des Permanganats tropfenweise unter Kühlung mit Eis zugefügt wurden. Die Oxydationsflüssigkeiten vom abgeschiedenen Braunstein dureh Abziehen mit dem Heber, Aufkochen, Filtriren und Pressen getrennt, wurden danu im Wasserbade auf ungefähr $2 \frac{1}{2}$ Liter eingeengt. Der bräunliche Schaleninhalt, der im wesentlichen Chitenidin enthalten muss, wurde behufs weiterer Oxydation allmälig mit einem Chrom-Sehwefelsäuregemisch von $430 \mathrm{~g}$ Chromsänre und $600 \mathrm{~g}$ Schwefelsäure in $3 \frac{1}{2}$ Liter Wasser versetzt.

Die auf Zusatz der ersten Partien eintretende braunrothe Farbe schlägt sehr rasch ius Smaragdgrïne um. Die Farbenändernng vollzieht sich bei den späteren Zugaben immer langsamer, bis sie endlich trotz mehrstïndigem Erwärmen am Wasserbade nicht mehr auftritt. Der Verlauf de: Oxydation ist also ganz so wie bei den anderen bisher untersuchten Chinabasen. Durch Zusatz einer kleinen Menge von Alkohol wurde die vollständige Reduction der Chromsäure herbeigefiibrt. Die rein grỉne Flüssigkeit ist in ganz derselben Weise, wie von $S \mathrm{kraup}$ angegeben, durch Eintragen in überschüssige Kalilauge von

1 Hes se, Annal. 1876, 183. 
Chrom befreit, durch Neutralisation und Eindampfen Kaliumsulfat abgeschieden, aus der alkoholischen Lösung der Kalisalze mit Schwefelsäure die organischen Säuren in Freiheit gesetzt, und durch Kochen mit Bleicarbonat in Bleisalze verwandelt worden. Die vom unlöslichen Bleisulfat, und überschüssigem Bleicarbonat getrennte Flüssigkeit schied, im Wasserbade eingedampft, beim Erkalten ein Bleisalz in Form eines dunklen pulverartigen Niederschlages (I) aus. Nach dessen Entfernung wurde das Filtrat bis zur Syrupconsistenz eingedampft, mit viel kaltem Wasser aufgenommen, vom neuerdings entstehenden Niederschlage getrennt, abermals stark eingeengt, und diese Manipulation solange fortgesetzt, bis der erhaltene Syrup vollkommen wasserlöslich war, und auch beim längeren Stehen keine Tribung mehr eintrat. Das in Wasser lösliche Bleisalz bis zur dicken Syrupconsistenz eingeengt, wog etwa $60 \mathrm{~g}$. In $150 \mathrm{~g}$ Wasser gelöst, und mit dem doppelten Volumen Alkohol versetzt, scheidet sich bei energischem Schütteln an den Wänden des Kolbens ein grünlichgelb gefärbtes Pflaster aus, das in Alkohol schwer, in Wasser mit Leichtigkeit löslich ist, und durch vier- bis fünfmalige Ausfällung mit Alkohol gereinigt wurde. Die genaue Bescbreibung dieser Fraction siehe in Abschnitt II.

Die bei der Ausscheidung und Reinigung dieses Bleisalzes erhaltenen alkoholischen Lösungen lassen sich nach dem Eindanpfen durch wiederholte Behandlung mit Alkohol zerlegen in kleine Mengen eines in diesem nicht löslichen Körpers, der identisch ist mit dem Bleisalze II, und in einen Syrup, der in Alkohol selrr leicht löslich, in Abschnitt III weiter beschrieben wird. Ausserdem fallen bei dieser Reinigung kleine Quantitäten eines in Wasser unlöslichen Bleisalzes ans, das sich bei der Untersuchung identisch mit dem in Abschnitt I beschriebenen erwies. I. Das in Wasser nnlösliche Bleisalz, welehes ich auf die oben erörterte Weise isolirt habe, wurde in verdünnter Salzsäure suspendirt, mit Schwefelwasserstoff zerlegt, vom Bleisulfid abfiltrirt, das Filtrat zum Syrup eingedampft und mit wenig verdïnnter Salzsäure aufgenommen. Nach dem Erkalten scheiden sich dünne prismatische Blättchen ab, die sehr Jeicht löslich, durch Verunreinigungen noch stark bräunlich gefärbt sind, und nach 
wiederholtem Umkrystallisiren aus wenig heissem Wasser unter Kochen mit Thierkohle von reingelber Farbe erhalten werden. Nach dem Lösen in viel kochendem Wasser scheiden sich beim Erkalten schwachgelbe Nadeln ab, die chlorfrei sind. Die Eigenschaften der chlorfreien Verbindungen entsprechen den Angaben von Skraup über die Chininsäure, die der chlorhältigen Substanz sind gleich jenen der Verbindung von Chininsäure mit Salzsäure. Die chlorfreie Verbindung ist krystallwasserfrei, und zeigt den Zersetzungs- und Schmelzpunkt um etwa $280^{\circ}$.

$0.1925 \mathrm{im}$ Exicator getrockneter Substanz geben: $0.4655 \mathrm{~g} \mathrm{CO}_{2}$ und $0.0918 \mathrm{~g} \mathrm{H}_{2} \mathrm{O}$.

\begin{tabular}{|c|c|}
\hline Gefunden & $\begin{array}{r}\begin{array}{c}\text { Berechnet für } \\
\mathrm{C}_{11} \mathrm{H}_{9} \mathrm{NO}_{3}\end{array} \\
\end{array}$ \\
\hline C $\ldots \ldots \ldots 64.72$ & 65.02 \\
\hline $\mathrm{H} \ldots \ldots \ldots \ldots 4.47$ & 4.34 \\
\hline
\end{tabular}

Es hat also wie nach dem Verlauf der Oxydation des Chitenin's von vornherein wahrscheinlich war, die Bildung von Chininsäure stattgefunden.

II. Das in Wasser leicht, in Alkohol nicht lösliche Bleisalz wurde in ungefähr $1 \frac{1}{2}$ Liter Wasser gelöst, im Wasserbade erwärmt, mit Schwefelwasserstoff zerlegt; das Filtrat vom Bleisulfid ist dann unter Zusatz von Salzsäure bis zur Syrupconsistenz eingedampft worden. Als nach mehrtägigem Stehen Krystallisation nicht erfolgte, wurde ein Kryställchen von salzsauerer Cincholoiponsäure aus Cinchonin eingeworfen, worauf tags darauf der Boden der Schale mit einer dicken Krystallkruste überzogen war, die aus grossen tafelförmigen Individuen bestand. Die Reinigung der Krystalle geschieht auch hier am leichtesten durch Waschen mit einem abgekiihlten Gemisch von Salzsäure und Alkohol, und Umkrystallisiren aus verdünnter Salzsäure.

Die erste Krystallisation betrug $14 g$.

Die Mutterlauge gab in der Winterkälte nach einwöchentlichem Stehen eine weitere Krystallisation von $5 g$. Dicse zweite Mutterlauge wurde zur Eutfernung der ïberschüssigen Salzsäure mehrmals zur Syrupconsistenz eingedampft. Nach Überführung in's Bleisalz und wiederholter Ausfällung desselben mit Alkohol 
konnten noch $2 g$ Krystalle gewonnen werden; der nicht unbedeutende Rest blieb dick syrupös.

Die Substanz bildet quadratische Tafeln mit schön ausge. bildetẹn Flächen, die von den Krystallen des Cincholoiponsäure-Chlorhydrates nicht zu unterscheiden sind. Sie ist chlorhältig; ibr Schmelzpunkt liegt zwischen 193-194 , im Moment der Verflüssigung tritt Gasentwicklung ein.

$0.191 g$ geben $0.3008 g \mathrm{CO}_{2}$ und $0.110 g \mathrm{H}_{2} \mathrm{O}$

$0.1557 g, 0.098 \mathrm{Ag} \mathrm{Cl}$

\begin{tabular}{|c|c|}
\hline Gefunden & $\mathrm{C}_{8} \mathrm{H}_{13} \mathrm{NO}_{4} \mathrm{HCl}$ \\
\hline C.......42.74 & 42.95 \\
\hline H. . . . . 6.34 & 6.26 \\
\hline Cl $\ldots \ldots \ldots 15.67$ & 15.88 \\
\hline
\end{tabular}

Aus diesen Daten folgt, dass die erhaltene Substanz dieselbe Zusammensetzung und dieselben Eigenschaften wie das Chlorhydrat der Cincholoiponsäure besitzt, das Skraup aus Cinchonin und Chinin, und Schniderschitsch aus Cinchonidin erhalten haben.

Meine Substanz erwies sich optisch gleichfalls activ. Eine $4 \%$ wässerige Lösung zeigte bei $l=200, d=1.0128, t=20$ eine Ablenknng von $+3^{\circ} 10^{\prime}$ und resultirt daraus $[\alpha]_{D}$ mit $+39.6^{\circ}$.

Schniderschitsch ${ }^{1}$ fand für sein aus Cinchonidin dargestelltes Chlorhydrat für eine $4 \%$ Lösung bei $l=200$ $d=1.0112, t=20$ eine Ablenkung von $+3.13^{\circ}$ daraus $+[\alpha]_{D}$ $40.2^{\circ}$.

Skraup fand für sein Chlorhydrat aus Cinchonin $\alpha$ mit $+3^{\circ} 1^{\prime}$, für jenes aus Chinin mit $2^{\circ} 43^{\prime}$.

Es liefert also das Chinidin ein Zerfallsproduct von denselben chemischen und physikalischen Eigenschaften wie die der anderen drei Chinaalkaloide.

Das krystallisirte Chlorhydrat konnte leicht in die freie Säure übergeführt werden, da nach entsprechender Verdünnung: der salzsaneren Lösung durch allmähliges Eintragen von Silberoxyd unschwer jener Moment fixirt werden kann, in dem nur-

1 Siehe die vorhergehende Mitthellung. 
mehr eine verschwindende Menge von Salzsäure vorhanden ist eine Methode, die sich deshalb als angenehm empfiehlt, weil von der Anwendung des Bleicarbonats und Schwefelwasserstoffs Umgang genommen ist.

Vom zusammengeballten Chlorsilber abfiltrirt und bis zum dünnen Syrup eingedampft, setzen sich nach einigem Stehen grosse, verwachsene Krystalle an, die nur zum Theil woblausgebildete Flächen zeigen.

Die lufttrockene Substanz schmilzt zwischen 126-127 unter gleichzeitiger Gasentwicklung, entwässert oder geschmolzen von derselben Erscheinung begleitet, zwischen $221^{\circ}-223^{\circ}$. Unter allmähliger Temperatur'steigerung schliesslich zwischen $120^{\circ}-125^{\circ}$ getrocknet gaben

$0.1878 g 0.0168 g \mathrm{H}_{2} \mathrm{O} \mathrm{ab}$.

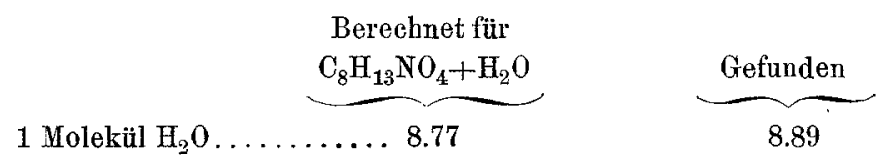

$0.171 g$ Trockensubstanz geben $0.3205 g \mathrm{CO}_{2}$ und $0.115 \mathrm{~g} \mathrm{H}_{2} \mathrm{O}$

$\underbrace{\text { Gefunden }}_{\mathrm{H} \ldots \ldots \ldots \ldots \ldots 1.12} \underbrace{\begin{array}{c}\text { Berechnet für } \\ \mathrm{C}_{8} \mathrm{H}_{13} \mathrm{NO}_{4}\end{array}}_{\begin{array}{r}51.26 \\ 6.95\end{array}}$

Eine $4 \%$ Lösung zeigte bei $l=200, d=1.0123, t=20$ eine Ablenkung von $2^{\circ} 29^{\prime}$, woraus sich $[\alpha]_{D}=30.9^{\circ}$ berechnet. Schniderschitsch fand unter analogen Bedingungen eine Ablenkung von $2^{\circ} 25^{\prime}$, S kra u p $2^{\circ} 24^{\prime}$ beziehlich $2^{\circ} 25^{\prime}$.

Es zeigt also auch die freie Cincholoiponsäure chemisch und physikalisch vollkommene Übereinstimmung mit der gleichzusammengesetzten Säure aus Cinchonin, Chinin und Cinchonidin.

III. Der in Alkohol leicht lösliche Theil des Oxydationsproductes zeigt eine dunkelbraune Färbung. Nachdem der Alkohol abdestillirt und der Destillationsrückstand mit Wasser wieder gelöst war, entstand auf Zușatz von Quecksilberchlorid ein reichlicher, lichtflockiger Niederschlag. Dieser sowohl, als auch dessen Filtrat wurden durch Schwefelwasserstoff zerlegt. Vom 
Quecksilbersulfid getrennt, zeigte das Filtrat des Niederschlages eine lichtgelbe Farbe, während das aus der Quecksilberverbindung eine mehr orangeartige besass. Beide lieferten eingedampft Krystallisationen.

Die aus dem Quecksilberniederschlag entstehende erwies sich als Chininsäure. Die Untersuchung der Krystallisation aus dem Filtrat des Quecksilberniederschlages hat bisher keine positiven Resultate ergeben. Tricarbopyridinsäure ist mit Bestimmtheit nicht vorhanden, ebenso Chininsäure. Dem eigenthiumlichen Geruche nach zu schliessen, der beim Erhitzen mit Ätzkalk auftritt, dürften in dieser Fraction Verbindungen enthalten sein, die der zweiten Chinidinhälfte entstanmen. Nach Beschaffung von mehr Material beabsichtige ieh eine genauere Untersuchung.

Durch die vorliegende Arbeit sind einige Schlüsse bezüglich der Constitution des Chinidin's gestattet.

Chinin und Chinidin können nur einen geringen Unterschied in ibrer Constitution haben. Die von Comstock und Königs auf ganz anderem Wege gemachten analogen Beobachtungen finden durch meine Arbeit eine auf so sprödem experimentellen Boden nicht unwichtige Bestätigung. Beide Alkaloide gehen durch Oxydation der ersten Hälfte ihrer Moleküle in Chininsäure über, beide besitzen ein Wasserstoffatom, das sich durch Säure radicale ersetzen lässt, und endlich enthalten sie einen gleichgegliederten Rest von acht Kohlenstoffatomen, auf den die Bildung der Cincholoiponsäure zurückzuführen ist. Der Unterschied in ihrer Constitntion kann nicht grösser sein als jener zwischen Cinchonin und Cinchonidin. Für die Beziehung zwischen den vier Alkaloiden wärees von grosser Wichtigkeit festzustellen, $o b$ ans dem Cinchonidin und Chinidin Körper gewonnen werden können, welche dem Cincholoipon aus Cinchonin und Chinin analog zusammengesetzt sind.

Eine Untersuchung darüber ist im Gange. 